\title{
Thermal Lens Temperature Scanning for Quantitative Measurements in Complex Fluids
}

\author{
J. H. Rohling, J. Mura, J. R. D. Pereira, A. J. Palangana, \\ A. N. Medina, A. C. Bento, M. L. Baesso, \\ Departamento de Física, Universidade Estadual de Maringá, \\ Av. Colombo 5790, CEP 87020-900, Maringá, PR, Brazil \\ and L. C. M. Miranda \\ Instituto Nacional de Pesquisas Espaciais, CEP 12227, Sâo José dos Campos, SP, Brazil
}

Received on 12 December, 2001

\begin{abstract}
In this work thermal lens spectrometry is applied to investigate the thermo-optical properties of complex fluids as a function of the temperature. The method is applied in poly(vinyl chloride), in polycarbonate and in lyotropic liquid crystals as the testing samples. The focus of the discussion will be in the temperature range where the phase transitions occur. The perspective of future studies in this area will be discussed.
\end{abstract}

\section{Introduction}

The ultimate goal in the study of the thermal and optical properties of complex fluids as a function of the temperature is the determination of the role played by these parameters in the material phase transitions [114]. It is well known that thermal conductivity $(K)$, thermal diffusivity $(D)$ and the coefficient of temperature of the refractive index $(d n / d T)$ present strong variation in their values when the temperature of the sample is close to the region where the phase transition is expected to occurs [1-7]. On the other hand, it is recognized that the precise determination of phase transition temperature is still an experimental challenge that needs to be accomplished. Conventional calorimetric methods demands the use of a reference sample and therefore a temperature lag between the tested sample and the reference may take place. This does not allow to locate the phase transition temperature precisely [1$5,12,13]$.

In the last two decades we have witnessed the development of a number of techniques for non-destructive characterization of the thermal, optical and structural properties of materials based upon the photothermal techniques [15, 16]. Despite this growing interest and the importance of the applications of these techniques to the complex fluid area [17-21], so far the photothermal measurements have been carried out mostly at near room temperature conditions. A restricted number of works has applied photothermal methods for different temperature studies in the complex fluids area. Mercuri and Marinelli $[1,2]$ employed a photopyroelet- ric method to investigate the critical behavior of the thermal parameters close to the liquid crystal phase transitions. Their experiments have been performed in thermotropic liquid crystal in the temperature range between $35{ }^{\circ} \mathrm{C}$ and $100{ }^{\circ} \mathrm{C}$. Recently, we have introduced Thermal Lens Spectrometry (TLS) to measure the thermo-optical properties of complex fluids as a function of the temperature [3-11]. The experiments have been performed in polymer samples, polyvinyl chloride and polycarbonate, and in pure and ferrofluid doped lyotropic liquid crystals. In these measurements the thermo-optical properties as a function of the temperature have been obtained. TLS is a very sensitive technique that has been proved to be a valuable method for investigating not only the complete thermal and spectroscopic properties of transparent materials, such as, glasses [22-33], liquid crystals [9-11,18] and polymers $[3-5,34]$, but also for the sensitive monitoring of the kinetics of fast chemical reactions [35], percolation in microemulsions [36], and dynamics of water-surfactant interaction [37]. This technique has the special ability of being a remote method and due to the fact that the necessary temperature to obtain the thermal lens signal is lower than $10^{-2} \mathrm{C}$, it allows a fine determination of the experimental parameters very close to the sample phase transition. One additional advantage of the TLS, especially for anisotropic samples, is the determination of the absolute value of $d n / d T$ for each sample orientation independently. The determination of $d n / d T$ as a function of the temperature and sample orientation is important because it contains information about sample thermal expansion coefficient, polarizability, refractive 
index, viscosity, etc [10]. The study of $d n / d T$ in liquid crystal is also attracting due to its unusual behavior. It has been observed that the refractive index change can produce either laser beam self-focusing and defocusing, depending on the sample orientation and temperature and also the laser beam polarization $[9,10,11]$.

In this work we discuss the use of the thermal lens (TL) technique for the measurements of the thermooptical properties of polymers and pure and ferrofluid doped lyotropic liquid crystals as a function of temperature. The focus of the discussion will be in the temperature range in which the phase transitions of these materials occur.

\section{Thermal lens Spectrometry}

Thermal Lens Spectrometry was first reported in 1964 by Gordan et al. [22]. Since then the technique has proved to be a valuable method to study transparent materials. The TL effect is induced when a laser beam passes through a material and the absorbed energy is converted into heat. The consequent change in the optical path length induced by a temperature rise will produce a lenslike optical element at the sample, the so called thermal lens effect. The propagation of a probe beam through the TL will then be affected resulting in a spreading or focusing of the beam center. By measuring this beam center intensity variation, the thermo-optical properties of the sample can be determined. The mode mismatched configuration has been shown to be the most sensitive experimental set up for the TL measurements. This arrangement uses two laser beams with different spot sizes at the sample position, as it is shown in Fig. 1.

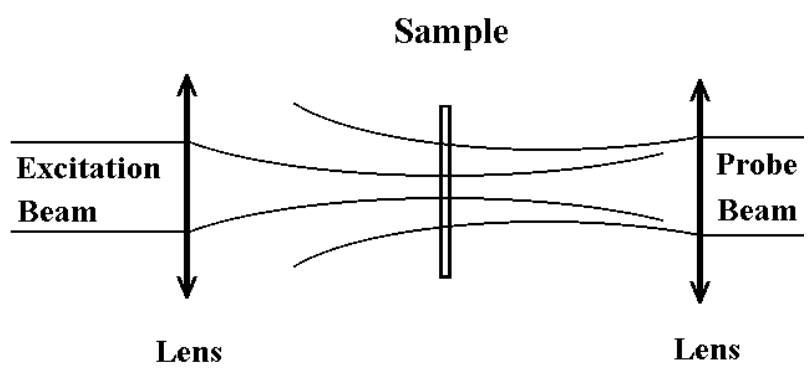

Figure 1. Probe beam and excitation beam in the mode mismatched TL configuration.

The theoretical treatment of the thermal lens effect takes into account the spherical aberration of the thermal lens and the whole optical length change with temperature is considered. The first step in the development of the model is to consider the heat source profile, $\mathrm{Q}(\mathrm{r})$, induced by the laser beam. $\mathrm{Q}(\mathrm{r})$ is proportional to the Gaussian intensity profile which can be expressed as $I_{e}(r)=\left(2 P_{e} / \pi \omega_{e}^{2}\right) \exp \left(-2 r^{2} / \omega_{e}^{2}\right)$, in which $\mathrm{P}_{e}$ is the excitation beam power and $\omega_{e}$ is the excitation beam waist at the sample position. The solution of the heat conduction equation depends on the employed boundary conditions. Shen et al. [38, 39] developed the infinitive aberrant model for the mode mismatched configuration. Using the conditions $\Delta T(r, 0)=0(r<\infty)$ and $\Delta T(\infty, t)=0(t>0)$, the temporal evolution of the temperature profile $\triangle T(r, t)$ induced by the TL in the sample is given by $[22,38]$ :

$$
\triangle T(r, t)=\frac{2 P_{e} A_{e}}{\pi c \rho \omega_{e}^{2}} \int_{0}^{t}\left[\frac{1}{1+\left(t^{\prime} / 2 t_{c}\right)}\right] \exp \left[\frac{-2 r^{2} / \omega_{e}^{2}}{1+\left(2 t^{\prime} / t_{c}\right)}\right] d t^{\prime}
$$

In Eq. (1) $\rho$ is the density, $c$ the specific heat, $A_{e}$ the optical absorption coefficient at the excitation beam wavelength and $t_{c}$ the characteristic thermal time constant, defined as:

$$
t_{c}=\frac{\omega_{e}^{2}}{4 D} ; D=\frac{K}{\rho c}
$$

where $D$ is the thermal diffusivity and $K$ is the thermal conductivity.

This temperature rise, which carries a Gaussian profile, induces a slight distortion in the probe beam wave front that can be associated with the optical path length change with respect to the axis as:

$$
\frac{\Phi \lambda_{p}}{2 \pi}=l_{0}\left(\frac{d s}{d T}\right)_{p}[\Delta T(r, t)-\Delta T(0, t)]
$$

in which $\Phi$ is the phase shift induced when the probe beam passes through the TL, $\lambda_{p}$ is the probe beam wavelength, $l_{0}$ is the sample thickness and $(d s / d T)_{p}$ is the temperature coefficient of the optical length at the probe beam wavelength.

Finally, using Fresnell diffraction theory, the probe beam intensity at the detector plane can be written as an analytical expression for absolute determination of the thermo-optical properties of the sample, as $[23,38,39]$ : 


$$
I(t)=I(0)\left[1-\frac{\theta}{2} \tan ^{-1}\left(\frac{2 m V}{\left((1+2 m)^{2}+V^{2}\right)\left(t_{c} / 2 t\right)+1+2 m+V^{2}}\right)\right]^{2}
$$

Where

$$
\theta=-\frac{P_{e} A_{e} l_{0}}{K \lambda_{p}}\left(\frac{d s}{d T}\right)_{p} ; V=\frac{Z_{1}}{Z_{c}} ; m=\left(\frac{\omega_{p}}{\omega_{e}}\right)^{2}
$$

In Eq. (4) $I(t)$ is the temporal dependence of the probe laser beam at the detector, $I(0)$ is the initial value of $I(t), \theta$ is the thermally induced phase shift of the probe beam after its passing through the sample, $\omega_{p}$ is the probe beam spot size at the sample, $Z_{c}$ is the confocal distance of the probe beam, $Z_{1}$ is the distance from the probe beam waist to the sample. We should mention that the parameter $d s / d T$ describes the whole optical path length change induced by the excitation beam, which means that for a solid materials it depends on several mechanisms such as the sample bulging during the illumination and also on the stressoptical coefficient. In the case of liquid sample we have $d s / d T=d n / d T$.

\section{Experimental}

TL measurements can be performed for both timeresolved and steady state mode. The time-resolved method permits the measurement of the development of the thermal lens in a short period of time, and the advantage of this procedure when compared with steady state mode is that it allows to measure the sample thermal diffusivity. The mode mismatched experimental set up for time resolved measurement is shown in Fig. 2. The experiment is performed in the following way: first, the probe beam is aligned, using mirror 5 , in order to have its center passing through the pinhole positioned in front of the detector; after that using mirror 2 the excitation beam is used to induce the thermal lens in the central part of the probe beam, inducing a consequent change in its intensity in the detector, photodiode 2. This signal variation is recorded by the oscilloscope and the data thus obtained is processed using least-square curve fitting. In the time resolved measurements, $\theta$ and $t_{c}$ are straightforwardly obtained from the fitting of the experimentally observed profile of the developing thermal lens to Eq. (4). In order to validate and evaluate the sensitivity of the proposed method we have also carried out complementary measurements of differential scanning calorimetry (DSC) and specific heat for the evaluation of the glass transition temperature.

The poly (vinyl chloride) (PVC) film used was a $200 \mu \mathrm{m}$ thick with $12 \mathrm{~mm}$ diameter disks. The PVC films were prepared using a 20,000 molar weight PVC powder (Aldrich). The films were cast from a $6.5 \%(\mathrm{w} / \mathrm{w}) 1,2$-di-chloroethane solution, at room temperature, over a flat clean glass substrate. The TL experiments were performed in the temperature range from $22{ }^{\circ} \mathrm{C}$ up to $70{ }^{\circ} \mathrm{C}$. For the polycarbonate, the tested sample used consisted of a $1.4 \mathrm{~mm}$ thick, 12 $\mathrm{mm}$ diameter discs of polysafe poly(carbonate) manufactured by Wilson Safety Products Co. The temperature range for the TL experiments was from $22{ }^{\circ} \mathrm{C}$ up to $170{ }^{\circ} \mathrm{C}$.

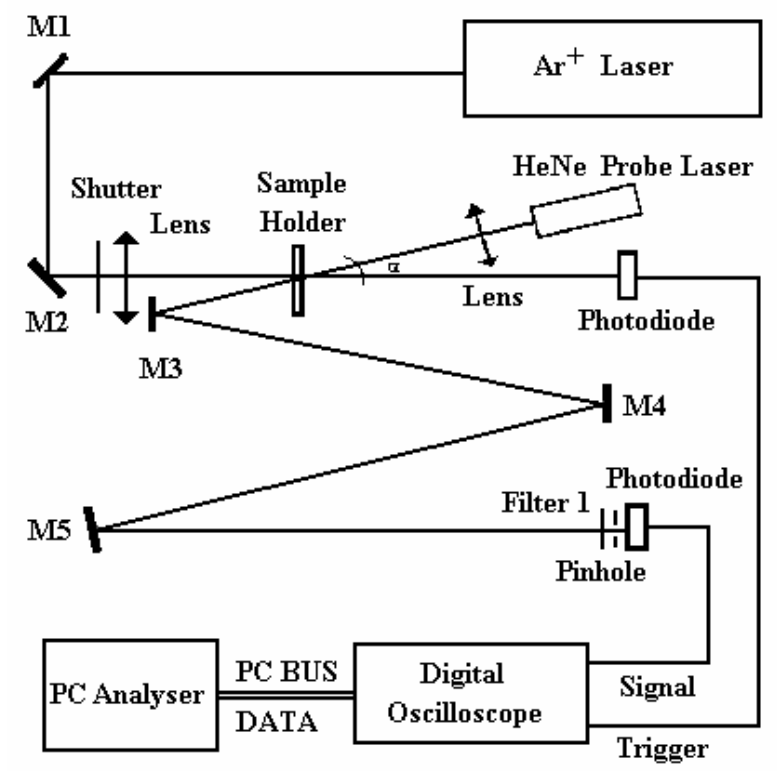

Figure 2. TL experimental set up.

The pure and ferrofluid doped lyotropic liquid crystal (LLC) samples were prepared with the following composition: potassium laurate (29.4 wt\%), decanol $(6.6 w t \%)$ and water(64wt\%). The phase sequences were determined by optical microscopy and conoscopic 
observations, which have shown that it was isotropic up to $15{ }^{\circ} \mathrm{C}$, calamitic nematic from $15{ }^{\circ} \mathrm{C}$ to $50{ }^{\circ} \mathrm{C}$ and isotropic again above $50^{\circ} \mathrm{C}$. The sample was placed in a quartz cuvette $0.5 \mathrm{~mm}$ thick. Using magnetic field the LLC director was aligned in parallel or perpendicular to the side walls. After that, the sample was positioned inside a hot stage (MK200) device. The measurements were performed as a function of the temperature in the range from $13{ }^{\circ} \mathrm{C}$ up to $54^{\circ} \mathrm{C}$.

\section{Thermal lens in polymers}

\section{IV.1 Room temperature study}

Polymers are carbonic compounds which, like glasses, present the required properties to be used as optical materials. When suitable for optical applications the lower cost of fabrication of these materials make them advantageous when compared to optical glasses. Therefore, it is important to determine the thermal lens effect in polymers since it is related to the change in the optical path length with temperature, $d s / d T$. The knowledge of this parameter is important when the material is used in optical system.

Fig. 3 shows a typical TL signal for the PVC sample at room temperature. The solid line corresponds to the data fitting of Eq. (4) to the TL experimental data leaving $\theta$ and $t_{c}$ as adjustable parameters. The values we have obtained were $\theta=(0.0791 \pm 0.0009)$ and $t_{c}=(5.50 \pm 0.06) \mathrm{ms}$. The corresponding value of the thermal diffusivity was $D=(1.29 \pm 0.03) \times 10^{-3} \mathrm{~cm}^{2} / \mathrm{s}$. This value agree with that reported in the literature for $\mathrm{PVC}$ at room temperature [7], namely, $1.2 \times 10^{-3} \mathrm{~cm}^{2} / \mathrm{s}$.

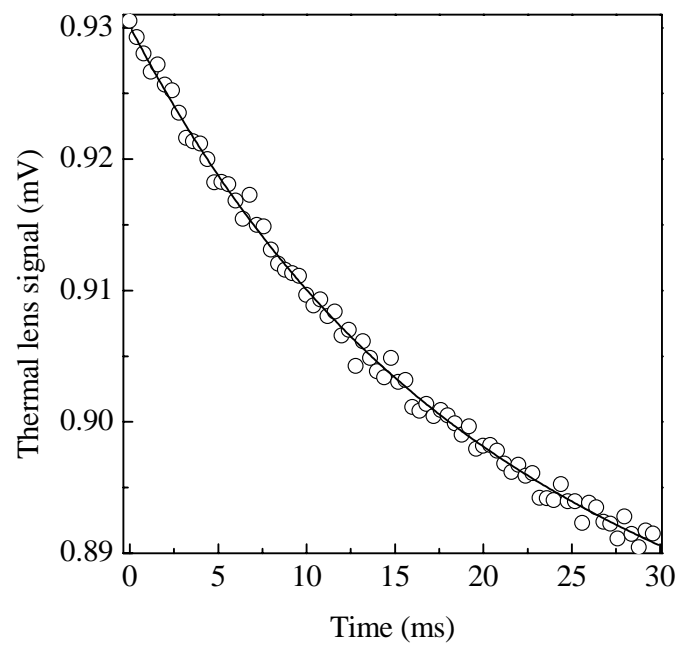

Figure 3. Typical transient TL signal for PVC sample for a pump beam power of $75 \mathrm{~mW}$ at room temperature. The solid line corresponds to the data fitting to Eq. (4).

We have performed additional measurements of specific heat and taken the mass density from the literature. By using the relation $K=\rho c D$ the thermal conductivity was obtained. At room temperature, the value we measured was $K=(1.87 \pm 0.03) \mathrm{mW} / \mathrm{cmK}$, which is close to the value reported in the literature, $1.6 \mathrm{~mW} / \mathrm{cmK}$.

Having the thermal conductivity, the measured value of $\theta / P=\left(1.1 \mathrm{~W}^{-1}\right), A_{e}=\left(0.1 \mathrm{~cm}^{-1}\right), k=(1.87$ $\mathrm{mW} / \mathrm{cmK}), \lambda_{p}=\left(6.328 \times 10^{-5} \mathrm{~cm}\right)$ and using Eq. (5) the value of $d s / d T$ was found to be $d s / d T=$ $\left(-0.62 \times 10^{-4} \mathrm{~K}^{-1}\right)$. For solid sample, the parameter $d s / d T$ determined by TL measurements can be written as [23]

$\frac{d s}{d T}=\left(n_{0}-1\right)(1+\nu) \alpha_{T}+\frac{d n}{d T}+\frac{1}{4} n_{0}^{3} Y \alpha_{T}\left(q_{11}+q_{12}\right)$,

in which $\alpha_{T}$ is the linear thermal expansion coefficient, $\nu$ is the Poisson ratio, $Y$ is the Young modulus, $q_{11}$ and $q_{12}$ are the stress optic coefficients, parallel and perpendicular to the direction of the laser beam propagation. Neglecting the last term of the above equation, that corresponds to about $10 \%$ of the total $d s / d T$, and using $n=1.546, d n / d T=-1.14 \times 10^{-4} \mathrm{~K}^{-1}$, and $\nu=0.38$ [7], we can estimate the value of $\alpha_{T}$ of our PVC sample as: $\alpha_{T}=6.9 \times 10^{-5} \mathrm{~K}^{-1}$. This results agrees with that from the literature [7], which is: $\left(6.8 \times 10^{-5} \mathrm{~K}^{-1}\right)$. For polycarbonate, the same procedure was adopted and we found $d s / d T=-0.21 \times 10^{-4} \mathrm{~K}^{-1}$.

\section{IV.2 Glass transition analysis}

The same procedure was adopted to carry out the experiments as a function of the temperature. Figures 4 and 5 show the resulting temperature dependence we obtained for the thermal diffusivity and the normalized thermal lens signal, $\theta$, of the PVC sample, respectively.

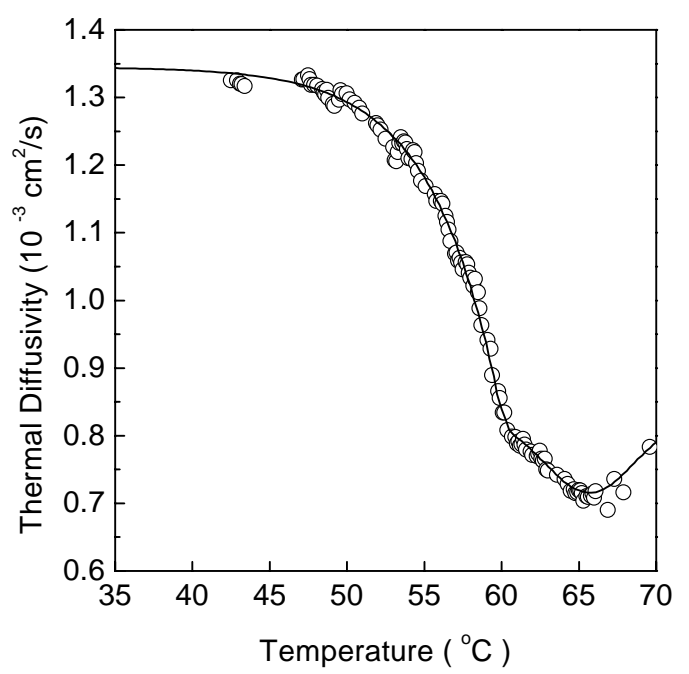

Figure 4. Thermal diffusivity of the PVC films as a function of temperature. 


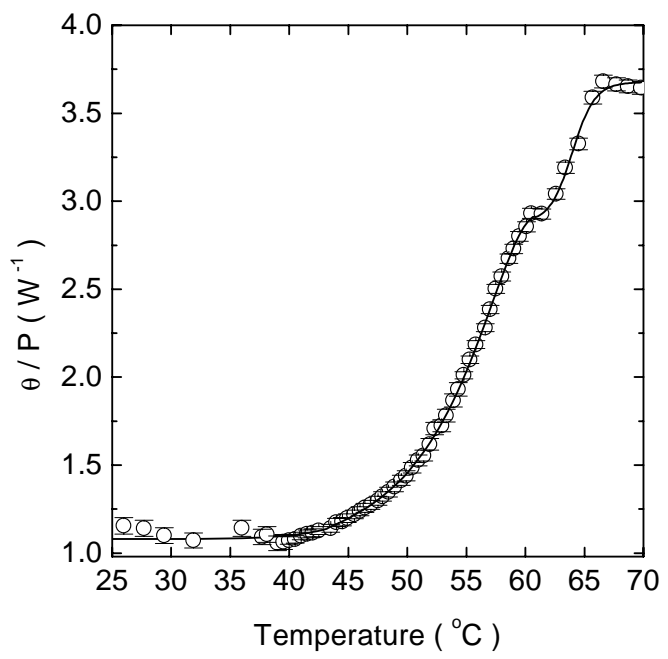

Figure 5. Thermal lens signal amplitude parameter of PVC as a function of the temperature.

To better understand the temperature dependence of both $D$ and $\theta / P$, we have also carried out DSC measurements. In Fig. 6a we present a typical DSC curve for our sample. This result indicates that the glass transition region of our PVC sample extends from 50 ${ }^{\circ} \mathrm{C}$ to about $67^{\circ} \mathrm{C}$ with a peak at $62.5^{\circ} \mathrm{C}$. From Figs. 4 and 5 we observe that both $D$ and $\theta / P$ show a marked change in their temperature dependence in this glass transition region. Between $50{ }^{\circ} \mathrm{C}$ to about $67{ }^{\circ} \mathrm{C}, D$ decreases by a factor of about 2 at the same time that $\theta / P$ experiences an increase of the order of 3.5.

The somewhat complex temperature dependence of $\theta / P$ may be better understood by looking at the temperature derivative of the solid line in Fig. 5, shown in Fig. 6b, which was obtained by using a combination of a Lorentzian function for the temperature range between $22{ }^{\circ} \mathrm{C}$ up to $60{ }^{\circ} \mathrm{C}$ and a logistic function

$$
\left(Y=A_{2}+\frac{\left(A_{1}-A_{2}\right)}{1+\pi\left(\frac{x}{x_{0}}\right)^{\nu}}\right)
$$

[4] for the temperatures above $60^{\circ} \mathrm{C}$. It tells us that, on increasing the temperature, $(1 / \theta)(d \theta / d T)$ increases, goes through a peak around $56{ }^{\circ} \mathrm{C}$, then decreases very sharply to a minimum at roughly $61^{\circ} \mathrm{C}$, to finally reach a maximum at $64{ }^{\circ} \mathrm{C}$, as shown in the upper curve. The comparison between the two plots in Fig. 6 is such that the first peak in curve (b), which occurs at $56{ }^{\circ} \mathrm{C}$, corresponds to the beginning of the glass transition as determined by the DSC curve, whereas the second peak of curve (b) corresponds to the inflection point of the DSC curve before reaching its minimum at $62.5^{\circ} \mathrm{C}$. We adopted this point as the glass transition temperature.

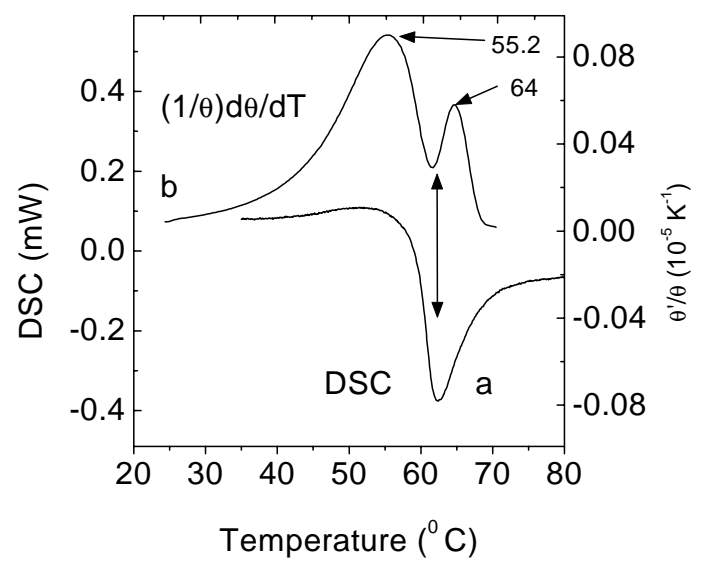

Figure 6. Temperature derivative of the TL signal(b) and DSC data(a) for PVC sample.

This result is indeed not as surprising as it may look at a first glance. In a DSC experiment one has a reference material, the sample to be probed, and a predetermined heating (or cooling) rate is imposed to the system for undergoing a given temperature excursion. A servo-system makes the sample to follow the temperature of the reference and the heating power difference between the sample and the reference is recorded. That is, since $d T / d t$ is fixed, one records essentially $d Q / d T$.

In Fig. 7 we show the resulting temperature dependence obtained for the thermal diffusivity and the TL signal parameter, $\theta / P$, for the polycarbonate sample, respectively. The DSC date is shown in curve (c). As performed for the PVC sample, in Fig. 8 we present the temperature derivative of $\theta / P$. For comparison, a plot of the temperature derivative of the DSC data is also presented in Fig. 8, curve (b). The existence of two minima in the DSC temperature derivative data seems to reflect the fact that the polycarbonate sample has two dominant phases. One phase corresponding to a glass transition temperature of about $143{ }^{\circ} \mathrm{C}$, and another one with $T_{g}$ of about $148{ }^{\circ} \mathrm{C}$. These two phases correspond to pure polycarbonate and to a PCA/ABS(acrylonitrile-butadienestyrene) blend. This ABS blend is used to improve the mechanical shock resistance of the polycarbonate used as safety eyeglasses. In temperature derivative of $\theta / P$ (Fig. 8a), in the temperature range between 140 ${ }^{\circ} \mathrm{C}$ and $160{ }^{\circ} \mathrm{C}$, the peaks exhibit a distinct correlation with those of the temperature derivative of DSC, Fig. 8b. The maxima occur at $144.8^{\circ} \mathrm{C}$ and $150{ }^{\circ} \mathrm{C}$, corresponding to the minima of the DSC temperature derivative. That is, the temperature dependence of $\theta / P$ apparently has more information than that contained in the DSC curve. 


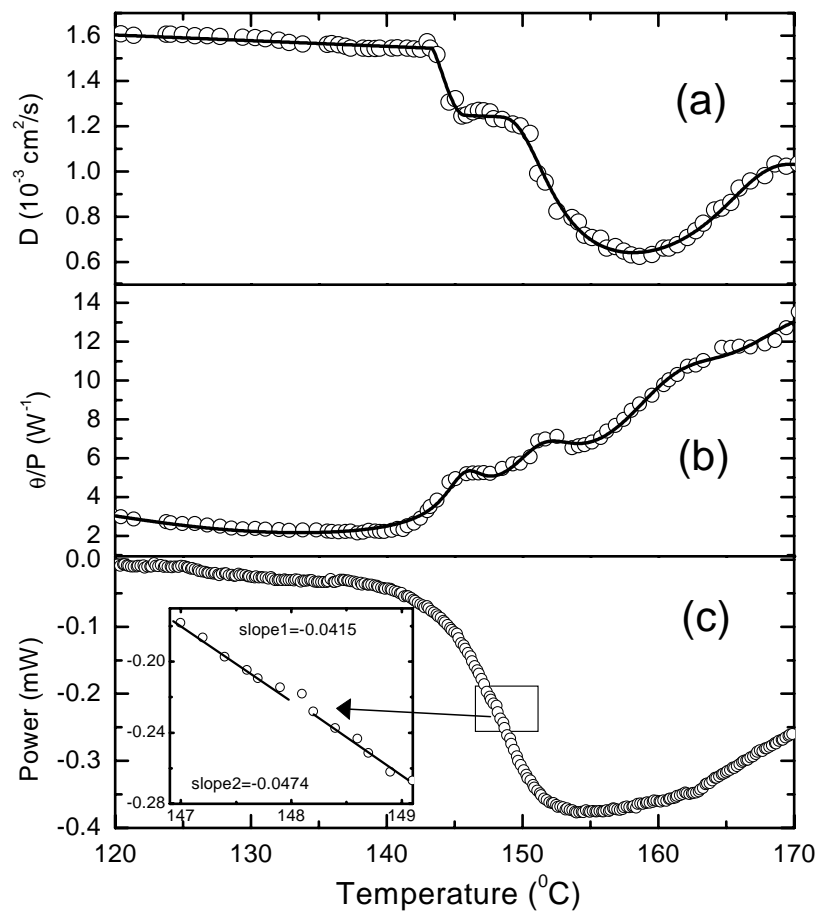

Figure 7. a: Temperature dependence of thermal diffusivity of the polycarbonate. The solid line represents the interpolation scheme for the data; $b$ : Temperature dependence of thermal lens amplitude for polycarbonate. The solid line is the interpolation obtained from the experimental data; c: Typical DSC curve for polycarbonate showing a wide glass transition from $140{ }^{\circ} \mathrm{C}$ to $155^{\circ} \mathrm{C}$. The inset shows the inflection point at $148^{\circ} \mathrm{C}$.

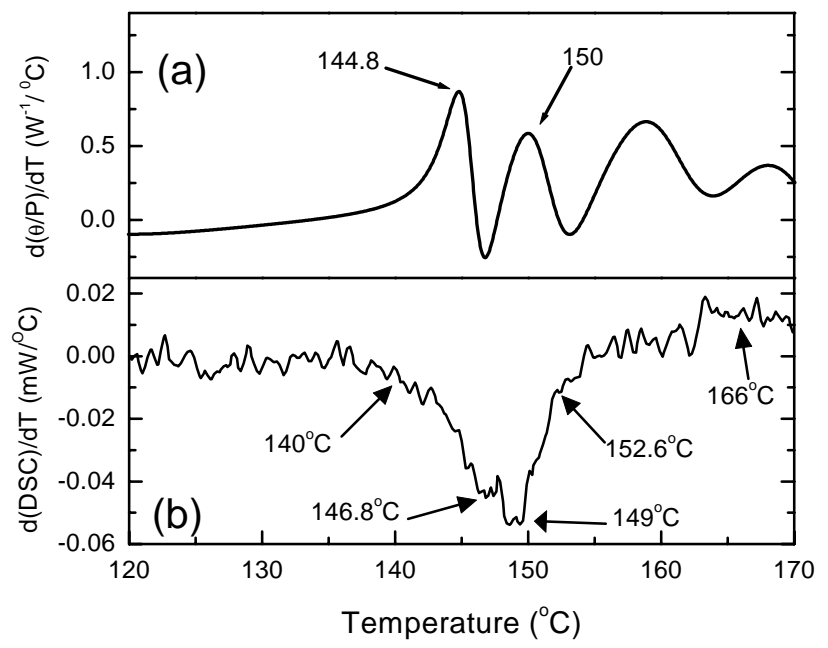

Figure 8. a: Temperature derivative of thermal lens signal amplitude obtained from the interpolated curve of Fig. 6b; b): Temperature derivative of the DSC data obtained from Fig. 6a.

The above results for PVC and polycarbonate indicate that the similarity between the behavior of $1 / \theta(d \theta / d T)$ and the DSC curve suggests that the TL technique can be used to perform an equivalent differ- ential scanning technique, namely, a differential thermal lens scanning, in which we set a given heating rate and measure the rate of change of the TL signal. The resulting signal of this technique would be $d \theta / d t=$ $(d \theta / d T)(d T / d t)$, that is, proportional to $d \theta / d T$, and can provide information regarding the onset of the glass transition.

\section{Thermal lens in lyotropic liq- uid crystal}

The similarity between lyotropic liquid crystal and biological membranes combined with the well known unique flow phenomena presented by liquid crystalline materials have attracted the attention of many researchers in the last few years. The study of this class of liquid crystals are of considerable interest due to the relative lack of detailed knowledge of their physical properties as compared to the thermotropic liquid crystals and considering the possibility for exploring these materials as sensing device elements.

The TL experiments have been performed for both orientation of the liquid crystal samples, i.e. paralell and perpendicular to the director alignment. It should be noted that the TL theoretical model was developed for an isotropic medium, while in the liquid crystal experiments, especially in the case of the planar geometry, the parameter $\theta$ has an effective value, defined as $\theta_{\| e}$. For homeotropic alignment there is a radial symmetry in the thermal lens profile, which means that the values of the measured parameters are related to the perpendicular orientation of the director. Here we denote $\theta$ as $\theta_{\perp}$.

Figures $9 \mathrm{a}$ and $9 \mathrm{~b}$ show the corresponding values of $\theta_{\| e}$ and $\theta_{\perp}$ (normalized to the laser power) for the pure LLC sample. For planar geometry the value of $\theta_{\| e}$ decreases from about 2 at $34{ }^{\circ} \mathrm{C}$ to 0.04 at $48.3^{\circ} \mathrm{C}$ and becomes negative between $48.5^{\circ} \mathrm{C}$ and $49.3^{\circ} \mathrm{C}$, coming back to a positive value above $49.3{ }^{\circ} \mathrm{C}$.

It follows from Eq. (6) that this inversion of the $\theta_{\| e}$ sign is a consequence of a change of sign of $d n / d T$. We note that this defocusing-self-focusing inversion was only observed for the planar geometry near the nematicisotropic phase transition. We attribute this inversion to the rate of change of the refractive index with respect to the temperature, namely, $d n / d T$. It is known that $d n / d T$ can be expressed as proportional to $A[\varphi-\beta]$ $[23,40,41]$, where $\varphi$ is the temperature coefficient of the electronic polarizability, $\beta$ is the thermal expansion coefficient, respectively, and $A$ is a constant that depends on the sample refractive index. In this relation $\varphi$ and $\beta$ are counteracting factors affecting the magnitude of $d n / d T$. In our results for the planar geometry, although the polarizability is comprised of two contributions of the refractive index change, parallel and perpendicular to the director, its value in the long axis orientation is 
dominant and probably controls the effective value of the probe beam phase shift, $\theta_{\| e}$.

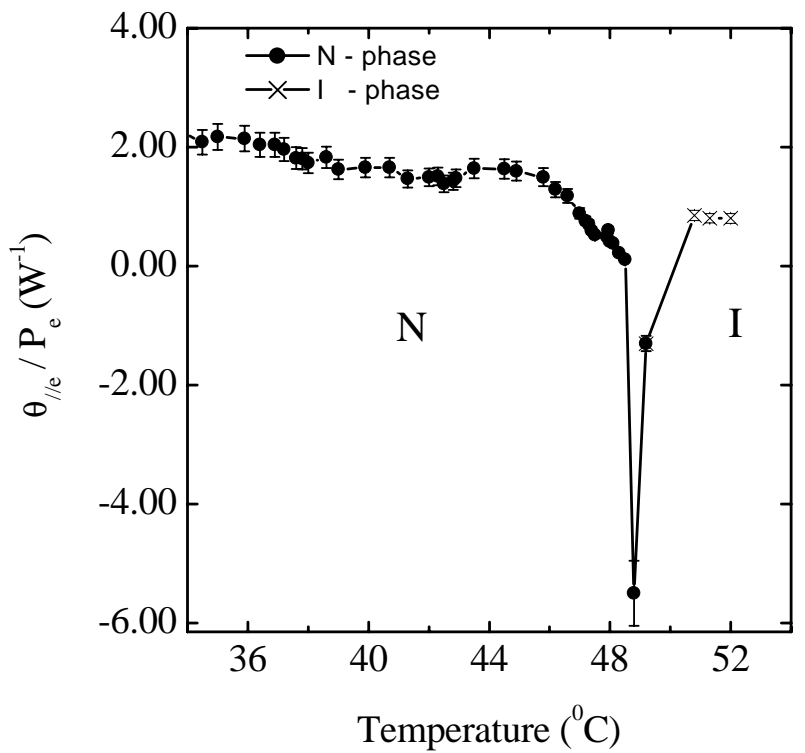

Figure 9a. Pure LC normalized probe beam phase shift $\theta_{\| e} / P_{e}$

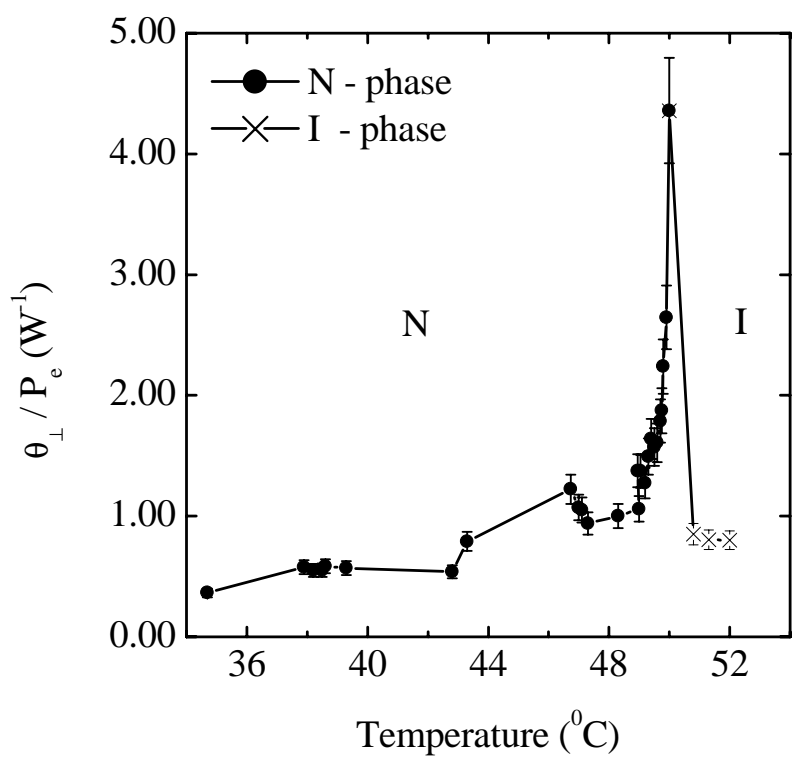

Figure 9b. Pure LC normalized probe beam phase shift $\theta_{\perp} / P_{e}$.

Therefore, a possible mechanism driving the observed inversion of $d n / d T$ in the director orientation is the increase in the $\varphi$ values in the long axis of the micelles, resulting from their higher electronic polarizability near the nematic-isotropic phase transition as compared to the nematic and isotropic phases. This agrees with the observation that, in the nematicisotropic phase transition the electronic polarizability is greatly enhanced in the axis parallel to the director [6]. The $\varphi$ value is associated to the electronic polarizing power $Z / a^{2}$, where $a$ is the distance between the dipole charges, $Z$. A decrease in the ratio $Z / a^{2}$ means a decrease in the atomic groups size producing a consequent increase in the value of these polarizing groups. This, in turn, suggests that the observed inversion in $d n / d T$ results from a change in the micelles shape, decreasing the distance between the dipole charges. This explanation is consistent with published X-ray diffraction measurements, showing the micelles shape change near the discotic nematic-isotropic phase transition in lyotropic liquid crystal [42].

For the homeotropic configuration the $\theta_{\perp}$ values increase with increasing temperature presenting a peak at $50{ }^{\circ} \mathrm{C}$, Fig. 9b. In this sample alignment the thermal expansion coefficient, $\beta$, dominates the observed change in $d n / d T$, which is negative in the whole temperature range investigated in this work.

We note in Fig. 9a, that the temperature of the peak of $\theta_{\| e}$ for the planar alignment is smaller than that of the peak in Fig. 9b for the homeotropic geometry. This difference may be attributed to the fact that the electronic polarizability increases fast in the beginning of the transition overcoming the possible changes in the thermal expansion values. After reaching the peak, the values of $\theta_{\| e}$ enters in a growing region until reaching the isotropic phase, indicating that in this temperature range the thermal expansion coefficient increases and dominates $d n / d T$. For the homeotropic geometry, as mentioned before, the thermal expansion coefficient dominates $d n / d T$ in the whole temperature range and becomes maximum just before the isotropic phase.

In Figures $10 \mathrm{a}$ and $10 \mathrm{~b}$ we show the temperature dependence of the normalized TL signal, $\theta$, for the ferrofluid doped sample. In this case the chosen temperature range included the isotropic-nematic transition which took place around $14{ }^{\circ} \mathrm{C}$. For the nematicisotropic transition the behavior of $\theta / P$ was similar to that occurred for the pure sample. However, the inversion in the $\theta / P$ values for the isotropic-nematic transition occurred for the director perpendicular orientation, indicating that the changes in the micellar shape is opposite as that for nematic-isotropic transition.

\section{Conclusion and future work}

In conclusion, in this paper we discuss the use of thermal lens technique to determine the thermo-optical properties of complex fluids as a function of the temperature. It is also discussed how the experimentally determined TL parameters can be used to locating the phase transition of these materials. The result showed the ability of the thermal lens technique to perform the measurements very close to the phase transition. As a future work, we propose that the technique could be adapted to develop a new method, called differential thermal lens scanning, especially designed for the investigation of the phase transition in complex fluids. 


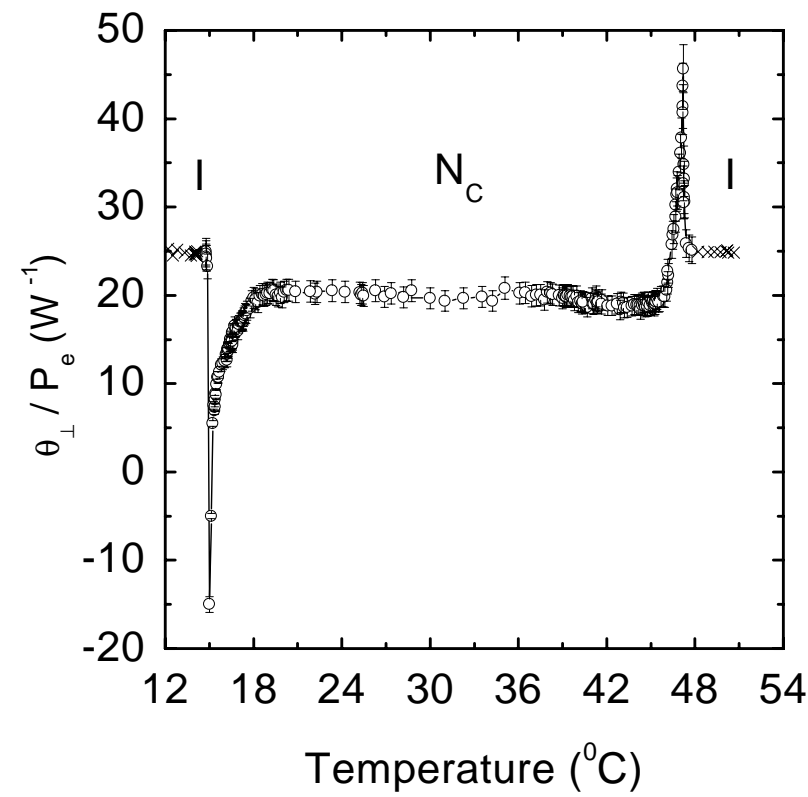

Figure 10a. Ferrofluid doped LC normalized probe beam phase shift $\theta_{\| e} / P_{e}$.

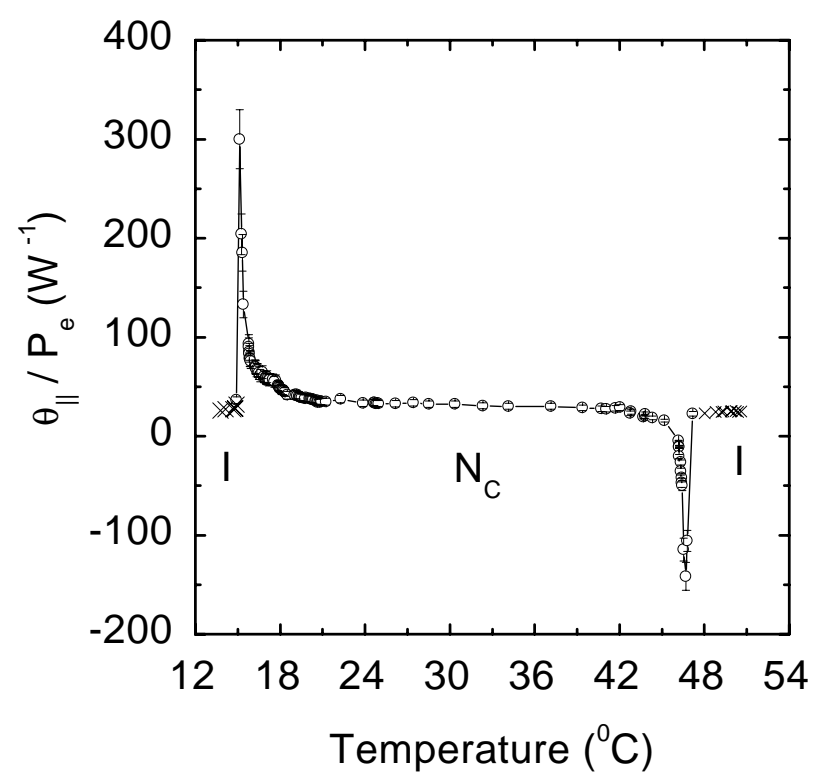

Figure 10b. Ferrofluid doped LC normalized probe beam phase shift $\theta_{\perp} / P_{e}$.

\section{Acknowledgements}

We are thankful to the Brazilian agencies CAPES, CNPq and Fundação Araucária for the financial support of this work.

\section{References}

[1] M. Marinelli, F. Mercuri, U. Zammit, and F. Scudieri, Phys. Rev. E 53, 701 (1996).

[2] F. Mercuri, M. Marinelli, U. Zammit, and F. Scudieri, Phys. Rev. E 57, 1 (1998).
[3] J.H. Rohling, A.N. Medina, A.C. Bento, J.R.D. Pereira, A.F. Rubira, M.L. Baesso, and L.C.M. Miranda, J. Phys. D: Appl. Phys. 34, 407 (2001).

[4] J.H. Rohling, A.N. Medina, A.C. Bento, J.R.D. Pereira, A.F. Rubira, M.L. Baesso, and L.C.M. Miranda, J. Appl. Phys. 89, 2220 (2001).

[5] J.H. Rohling, A.N. Medina, J.R.D. Pereira, A.F. Rubira, A.C. Bento, L.C.M. Miranda, and M.L. Baesso, Analytical Sciences 17, s103 (2001).

[6] G. Vertogen and W. H. de Jeu, Thermotropic Liquid Crystal, (Springer Verlag, Berlin, 1988).

[7] J. Bandrup and E.H. Immerght, Polymer Handbook, 3rd ed., pp. V/1ed. (Wiley, New York, 1989).

[8] M.L. Baesso, J. R. D. Pereira, A.C. Bento, A. J. Palangana, A.M. Mansanares, and L. R. Evangelista, Braz. J. Physics 28, 359 (1998).

[9] J.R.D. Pereira, A.J. Palangana, A.M. Mansanares, E.C. da Silva, A.C. Bento, and M.L. Baesso, Phys. Rev. E, 61, 5410 (2000).

[10] J.R.D. Pereira, A.M. Mansanares, A.J. Palangana, M.L. Baesso, A.A. Barbosa, and P.R.G. Fernandes, Phys. Rev. E, 64, 062701 (2001).

[11] J.R.D. Pereira, A.M. Mansanares, A.J. Palangana, and M.L. Baesso, Phys. Rev. E, 6410, 012701 (2001).

[12] G. Hohne, W. Hemminger, and H.-J. Flammershein, in: Differencial Scanning Calorimetry: A Introduction for Practioners. (Springer Verlag, 1996).

[13] J.H. Rohling, A.N. Medina, A.C. Bento, J.R.D. Pereira, M.L. Baesso, L.C.M. Miranda, S.M. Lima, and T. Catunda, Analytical Sciences 17, s106 (2001).

[14] J.R.D. Pereira, A.J. Palangana, A.M. Mansanares, E.C. da Silva, A.C. Bento, and M.L. Baesso, Analytical Sciences, 17, s175 (2001).

[15] A. Rosencwaig, Photoacoustics and Photoacoustic Spectroscopy (Wiley, New York, 1980).

[16] H. Vargas and L.C.M. Miranda, Phys. Rep. 161, 43 (1988).

[17] A. Lachaine and P. Poulet, Appl. Phys. Lett. 45, 953 (1984).

[18] A.C. Bento, A.J. Palangana, L.R. Evangelista, M.L. Baesso, J.R.D. Pereira, E.C. da Silva, and A.M. Mansanares, Appl. Phys. Lett. 68, 3371 (1996).

[19] J.R.D. Pareira, A.M. Mansanares, A.J. Palangana, and M.L. Baesso, Mol. Cryst. Liq. Cryst. A, 332, 3079 (1999).

[20] W.L.F. dos Santos, M.F. Porto, E.C. Muniz, L.Olenka, M.L. Baesso, A.C. Bento, and A.F. Rubira, J. Appl. Polymer Science, 77, 289 (2000).

[21] L.Olenka, E.N. Nogueira, W.L.F. dos Santos, E.C. Muniz, A.F. Rubira, A.N. Medina, L.P. Cardoso, L.C. Miranda, M.L. Baesso, and A.C. Bento, J. Phys. D: Appl. Phys. 34, 2248 (2001).

[22] J.P. Gordon, R.C.C. Leite, R.C. Morre, S.P.S. Porto, and J.R. Whinnery, J. Appl. Phys. 36, 3 (1965). 
[23] M.L. Baesso, J. Shen, and R.D. Snook. J. Appl. Phys. 75, 3732 (1994).

[24] M.L. Baesso, A.C. Bento, A.R. Duarte, A.M. Neto, and L.C.M. Miranda, J. Appl. Phys. 85, 8112 (1999).

[25] M. L. Baesso, J. Shen, and R. D. Snook, Chem. Phys. Lett. 197, 255 (1992).

[26] M.L. Baesso, A.C. Bento, A.A. Andrade, T. Catunda, J.A. Sampaio, and S. Gama, J. Non-Cryst. Solids 219, 165 (1997).

[27] M. L. Baesso, A. C. Bento, A. A. Andrade, J. A. Sampaio, E. Pecoraro, L. A. O. Nunes, T. Catunda, and S. Gama, Phys. Rev. B 57, 10545 (1998).

[28] S.M. Lima, T. Catunda, R. Lebullenger, A.C. Hernandes, M.L. Baesso, A.C. Bento, and L.C.M. Miranda, Phys. Rev. B 60, 15173 (1999).

[29] A. A. Andrade, T. Catunda, R. Lebullenger, A.C. Hernandes, and M.L. Baesso, J. Opt. Soc. of Am. B 16, 395 (1999).

[30] A. A. Andrade, T. Catunda, R. Lebullenger, A.C. Hernandes, and M.L. Baesso, Elect. Lett. 34, 117 (1998).

[31] S.M. Lima, T. Catunda, M.L. Baesso, L.D. Vila, Y. Messadeq, E.B. Stucchi, and S.J.L. Ribeiro, J. NonCryst. Solids 247, 222 (1999).
[32] S.M. Lima, J.A. Sampaio, T. Catunda, R. Lebullenger, A.C. Hernandes, M.L. Baesso, A.C. Bento, and F.C.G. Gandra, J. Non-Cryst. Solids 257, 337 (1999).

[33] S.M. Lima, A.A. Andrade, T. Catunda, R. Lebullenger, A.C. Hernandes, and M.L. Baesso, Appl. Phys. Lett. 78, 3220 (2001).

[34] R. D. Snook, R. D. Lowe, and M. L. Baesso, Analyst 23, 587 (1998).

[35] M. Franko and C.D. Tran, Rev. Sci. Instrum. 62, 2438 (1991).

[36] M.S. Baptista and C.D. Tran, J. Phys. Chem. B 101, 4209 (1997).

[37] M.S. Baptista and C.D. Tran, J. Phys. Chem. 99, 12952 (1995).

[38] J. Shen, R. D. Lowe, and R. D. Snook, Chem. Phys. 165, 385 (1992).

[39] J. Shen, M. L. Baesso, and R. D. Snook, J. Appl. Phys. 75, 3738 (1994).

[40] M. Sparks, J. Appl. Phys. 42, 5029 (1971).

[41] L. Prod'homme, Phys. and Chem. of Glasses 1, 119 (1961).

[42] Y. Galerne, A. M. Figueiredo Neto, and L. Liebént, Phys. Rev. A 31, 4047 (1985). 